\title{
Correlated Coulomb drag in capacitively coupled quantum-dot structures
}

\author{
Kaasbjerg, Kristen; Jauho, Antti-Pekka
}

Published in:

Physical Review Letters

Link to article, DOI:

10.1103/PhysRevLett.116.196801

Publication date:

2016

Document Version

Publisher's PDF, also known as Version of record

Link back to DTU Orbit

Citation (APA):

Kaasbjerg, K., \& Jauho, A-P. (2016). Correlated Coulomb drag in capacitively coupled quantum-dot structures. Physical Review Letters, 116, [196801]. https://doi.org/10.1103/PhysRevLett.116.196801

\section{General rights}

Copyright and moral rights for the publications made accessible in the public portal are retained by the authors and/or other copyright owners and it is a condition of accessing publications that users recognise and abide by the legal requirements associated with these rights.

- Users may download and print one copy of any publication from the public portal for the purpose of private study or research.

- You may not further distribute the material or use it for any profit-making activity or commercial gain

- You may freely distribute the URL identifying the publication in the public portal

If you believe that this document breaches copyright please contact us providing details, and we will remove access to the work immediately and investigate your claim. 


\title{
Correlated Coulomb Drag in Capacitively Coupled Quantum-Dot Structures
}

\author{
Kristen Kaasbjerg ${ }^{*}$ and Antti-Pekka Jauho \\ Center for Nanostructured Graphene (CNG), Department of Micro- and Nanotechnology, \\ Technical University of Denmark, DK-2800 Kgs. Lyngby, Denmark
}

(Received 4 January 2016; published 10 May 2016)

\begin{abstract}
We study theoretically Coulomb drag in capacitively coupled quantum dots (CQDs) — a bias-driven dot coupled to an unbiased dot where transport is due to Coulomb mediated energy transfer drag. To this end, we introduce a master-equation approach that accounts for higher-order tunneling (cotunneling) processes as well as energy-dependent lead couplings, and identify a mesoscopic Coulomb drag mechanism driven by nonlocal multielectron cotunneling processes. Our theory establishes the conditions for a nonzero drag as well as the direction of the drag current in terms of microscopic system parameters. Interestingly, the direction of the drag current is not determined by the drive current, but by an interplay between the energydependent lead couplings. Studying the drag mechanism in a graphene-based CQD heterostructure, we show that the predictions of our theory are consistent with recent experiments on Coulomb drag in CQD systems.
\end{abstract}

DOI: $10.1103 /$ PhysRevLett.116.196801

Electronic systems brought into close proximity may exhibit Coulomb drag [1,2]: a current in one system induces a current (or a voltage) in a nearby undriven system. Importantly, the effect arises solely due to Coulomb interactions between the charge carriers in the two systems. Coulomb drag has been studied extensively in bulk two-dimensional systems, both experimentally [3-5] and theoretically [6-9], and has recently experienced a revival in one-dimensional systems [10-14] and graphene heterostructures [15-20].

In mesoscopic systems with broken translational invariance, e.g., quantum point contacts or quantum dots (QDs), momentum is not a good quantum number as in extended systems. Instead of momentum transfer, it is more natural to view mesoscopic Coulomb drag [21-25] as an interaction mediated energy transfer between the drive and the drag system. Such energy-transfer drag plays a central role in, for example, quantum measurements where a detector and a system exchange energy in a measurement on the system [26]. In this case, the drag can either constitute the signal in the detector generated by the measured quantum noise in the system [27-29], or be a disturbance in the system due to the measurement [30,31], i.e., detector backaction.

In addition to energy transfer, Coulomb drag in capacitively coupled QDs (CQDs) relies on an asymmetry in the drag system [25]. This has been demonstrated in coupled double quantum dots [32], and recently in coupled single QDs [33,34], where the asymmetry originates from the couplings to the leads. In the latter, Coulomb-drag effects beyond conventional mesoscopic QD drag [25] were reported [33]. Not only are such effects of fundamental scientific interest, but they may also be important for the performance of thermoelectric CQD devices [35-38].
In this work we introduce a theoretical framework for the description of Coulomb drag in CQDs taking into account higher-order tunneling (cotunneling) processes, and thereby going beyond conventional QD drag [25]. We uncover a drag mechanism driven by nonlocal correlated multielectron cotunneling processes where energy transfer is mediated by bias-induced switching of the CQD states. At the triple points of the CQD charge stability diagram [39] sketched in Fig. 1(a), it resembles a stochastic ratchet (a)

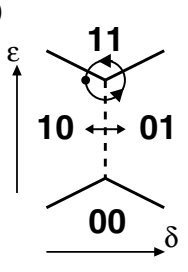

(b)

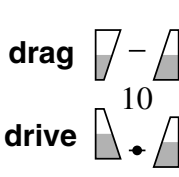

(c)

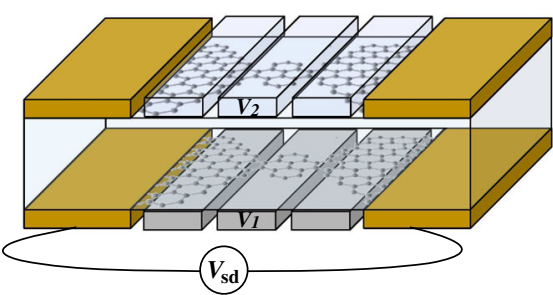

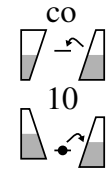
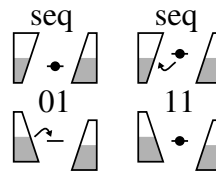
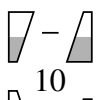

FIG. 1. (a) Charge stability diagram of two capacitively coupled QDs as a function of their gate detuning $\delta=V_{2}-V_{1}$ and common gate $\epsilon=V_{1}+V_{2}$. (b) Sequence of sequential and cotunneling processes underlying the drag mechanism in the vicinity of the triple points [closed circle in (a)]. Away from the triple points, the drag is driven by cotunneling only [arrow in (a)]. Energy-dependent lead couplings are essential for the mechanism to induce a directional current in the drag system. (c) Illustration of a graphene-based CQD heterostructure with two QDs defined in stacked graphene layers separated by a thin isolating dielectric [33]. A series of top and bottom gates control the potentials on the quantum dots $\left(V_{1 / 2}\right)$ and their adjacent graphene leads. 
mechanism which, like charge pumping mechanisms [40], results in drag via excursions [in state space; see Fig. 1(b)] around the triple points. Our theory pinpoints the conditions for drag in terms of microscopic quantities and shows that the direction of the drag current is independent on the drive current and exhibits a nontrivial dependence on the lead couplings in the drag system.

We demonstrate the rich properties of the drag mechanism by studying drag in the graphene-based CQD structure illustrated in Fig. 1(c). Such experimentally realizable graphene-based QD structures are unique due to their large tunability [41-45], large interdot charging energies [33], and built-in graphene leads. We envision structures in which local gating allows us to control the chemical potentials of the lead regions $[46,47]$ and create, e.g., $p$-QD- $n$ junctions across the individual QDs. As we demonstrate below, this opens the opportunity to control the direction of the drag current. Finally, we elaborate on the role of the drag mechanism in the recently observed Coulomb drag in a graphene-based CQD heterostructure [33].

General model and theory.-We consider a generic (spinless) model for two capacitively coupled QDs-a biased drive $(i=1)$ and an unbiased drag $(i=2)$ QDwith one level each, $H_{\mathrm{CQD}}=\sum_{i} \varepsilon_{i} n_{i}+U_{12} n_{1} n_{2}$, where the dot levels are controlled by gate voltages $\varepsilon_{i}=-e V_{i}$, $n_{i}=d_{i}^{\dagger} d_{i}$ is the dot occupation, and $U_{12}=e^{2} / 2 C$ is the capacitive interdot Coulomb interaction. The dots are coupled to separate sets of source and drain contacts, $H_{\alpha}=\sum_{k} \xi_{\alpha k} c_{\alpha k}^{\dagger} c_{\alpha k}, \quad \xi_{\alpha k}=\varepsilon_{k}-\mu_{\alpha} \quad\left(\alpha=L_{i}, R_{i} ; \mu_{L_{1} / R_{1}}=\right.$ $\pm e V_{\text {sd }} / 2+\mu_{0}$ and $\left.\mu_{L_{2} / R_{2}}=\mu_{0}\right)$, via tunnel Hamiltonians $H_{T}=\sum_{\alpha k} t_{\alpha k} c_{\alpha k}^{\dagger} d_{i}+$ H.c.. In contrast to the usual wideband approximation where the lead couplings are assumed constant, we here consider energy-dependent couplings $\Gamma_{\alpha}(\varepsilon)=2 \pi \rho_{\alpha}(\varepsilon)\left|t_{\alpha}(\varepsilon)\right|^{2}$, where $\rho_{\alpha}$ is the density of states (DOS) in lead $\alpha$ and $t_{\alpha}$ is the tunnel coupling. Like in conventional QD drag [25,33], this is the key ingredient for the drag mechanism described below.

We describe the transport through the drive and drag dots with a master equation approach valid for $k_{B} T \gtrsim \Gamma_{\alpha}$ [48]. The occupation probabilities $p_{m}$ for the CQD states, $|m\rangle=\left|n_{1} n_{2}\right\rangle \in\{|00\rangle,|10\rangle,|01\rangle,|11\rangle\}$, are determined by the rate equations

$$
\dot{p}_{m}=-p_{m} \sum_{n \neq m} \Gamma_{m n}+\sum_{n \neq m} p_{n} \Gamma_{n m},
$$

which together with the normalization condition $\sum_{m} p_{m}=1$ are solved for the steady-state probabilities, i.e., $\dot{p}_{m}=0$.

The rates for tunneling-induced transition between the states are obtained from the generalized Fermi golden rule [48],

$$
\Gamma_{m n}=\frac{2 \pi}{\hbar} \sum_{i^{\prime} f^{\prime}} W_{i^{\prime}}|\langle f|T| i\rangle|^{2} \delta\left(E_{f}-E_{i}\right) .
$$

Here, $|i / f\rangle=|m / n\rangle \otimes\left|i^{\prime} / f^{\prime}\right\rangle$ are products of QD and lead states, the sum is over possible initial $\left|i^{\prime}\right\rangle$ and final $\left|f^{\prime}\right\rangle$ states of the leads, $W_{i^{\prime}}$ is the probability for the initial lead state $\left|i^{\prime}\right\rangle$, and $T=H_{T}+H_{T} G_{0} H_{T}+\ldots$ is the $T$ matrix with $G_{0}=1 /\left(E_{i}-H_{0}\right)$ denoting the Green function in the absence of tunneling, i.e., $H_{0}=H_{\mathrm{CQD}}+\sum_{\alpha} H_{\alpha}$. The correlations between the occupations of the QDs are fully accounted for in $G_{0}$, which is treated exactly.

To lowest order in the tunneling Hamiltonian, the transitions between the states are given by sequential tunneling processes with rates

$$
\begin{aligned}
& \Gamma_{m, 11}^{\alpha}=\hbar^{-1} \Gamma_{\alpha}\left(\Delta_{m, 11}\right) f_{\alpha}\left(\Delta_{m, 11}\right), \\
& \Gamma_{m, 00}^{\alpha}=\hbar^{-1} \Gamma_{\alpha}\left(\Delta_{00, m}\right)\left[1-f_{\alpha}\left(\Delta_{00, m}\right)\right], \\
& \Gamma_{00, n}^{\alpha}=\hbar^{-1} \Gamma_{\alpha}\left(\Delta_{00, n}\right) f_{\alpha}\left(\Delta_{00, n}\right), \\
& \Gamma_{11, n}^{\alpha}=\hbar^{-1} \Gamma_{\alpha}\left(\Delta_{n, 11}\right)\left[1-f_{\alpha}\left(\Delta_{n, 11}\right)\right],
\end{aligned}
$$

where $m, n \in\{10,01\}, f_{\alpha}$ is the Fermi function in lead $\alpha$, and $\Delta_{m n}=E_{n}-E_{m}$.

The next-to-leading order term in the $T$ matrix gives rise to elastic and inelastic cotunneling through the individual QDs [49-51]. In addition, we identify a nonlocal cotunneling process mediated by the capacitive interdot coupling. This is a correlated two-electron tunneling event in which the CQD switches between the $10 \leftrightarrow 01$ states in one coherent process. The rate for nonlocal cotunneling processes which transfer an electron from lead $\alpha$ to lead $\beta$ is given by

$$
\begin{aligned}
\Gamma_{m n}^{\alpha \beta}= & \int \frac{d \varepsilon}{2 \pi \hbar} \Gamma_{\alpha}\left(\varepsilon+\Delta_{m n}\right) \Gamma_{\beta}(\varepsilon) f_{\alpha}\left(\varepsilon+\Delta_{m n}\right)\left[1-f_{\beta}(\varepsilon)\right] \\
& \times\left|\frac{1}{\varepsilon+\Delta_{11, n}}-\frac{1}{\varepsilon+\Delta_{m, 00}}\right|^{2}
\end{aligned}
$$

where $m, n \in\{10,01\}$ and the terms in the last line account for the energy of the virtually occupied intermediate $00 / 11$ states. To evaluate the cotunneling rates at finite temperature and bias, we have generalized the commonly applied regularization scheme $[52,53]$ to the situation with energydependent lead couplings [54].

From the solution to the master equation (1), the currents in the various leads are obtained as

$$
I_{\alpha}=-e \sum_{m n} p_{m}\left(\Gamma_{m n}^{\rightarrow \alpha}-\Gamma_{m n}^{\alpha \rightarrow}\right)
$$

where $\Gamma^{\rightarrow \alpha}\left(\Gamma^{\alpha \rightarrow}\right)$ denotes the rate for processes that transfer an electron into (out of) lead $\alpha$, and the drive and drag currents are defined as $I_{\text {drive }}=I_{L_{1}}=-I_{R_{1}}$ and $I_{\text {drag }}=I_{L_{2}}=-I_{R_{2}}$, respectively.

Drag mechanism.-In the following, we focus on the regime of low bias on the drive $\mathrm{QD}, e V_{\text {sd }} \lesssim U_{12}$, where the 
conventional drag mechanism [25] is suppressed. Fixing the gate voltages to, e.g., the point below the 10,11 degeneracy line at the upper triple point in Fig. 1(a), a finite bias on the drive QD opens for the sequence of transitions illustrated in Fig. 1(b),

$$
|10\rangle \stackrel{\text { co }}{\leftrightarrow}|01\rangle \stackrel{\text { seq }}{\leftrightarrow}|11\rangle \stackrel{\text { seq }}{\rightarrow}|10\rangle .
$$

For $e V_{\mathrm{sd}}>\left|\Delta_{10,01}\right|,\left|\Delta_{01,11}\right| \gg k_{B} T$, the two first transitions are open in both directions, whereas the third transition is only open in the forward direction because the drag QD is unbiased. In addition to a drive current, this may induce a drag current via steps where the drag QD is repeatedly filled and emptied. This is possible via the first step alone (cotunneling-only), or through the full sequence (cotunneling-assisted drag). The two mechanisms govern the drag, respectively, away from and at the triple points [cf. Fig. 1(a)]. Note that the nonlocal cotunneling process is instrumental in both cases.

In order to generate a drag current, the drag QD must be filled and emptied at preferentially separate leads. This requires an asymmetry in the drag system. To identify the exact conditions, we expand the lead couplings around the equilibrium chemical potentials $\mu_{0}, \Gamma_{\alpha}(\varepsilon) \approx \Gamma_{\alpha 0}+\xi \partial \Gamma_{\alpha}$, where $\xi=\varepsilon-\mu_{0}, \Gamma_{\alpha 0}=\Gamma_{\alpha}\left(\mu_{0}\right)$, and $\partial \Gamma_{\alpha}=\partial \Gamma_{\alpha} /\left.\partial \varepsilon\right|_{\varepsilon=\mu_{0}}$. Along the 10,01 degeneracy line where $\Delta_{10,01}=0$, and in the nonlinear regime $e V_{\text {sd }} \gg k_{B} T$ (but still $e V_{\text {sd }}<U_{12}$ ) where the transport in the drive QD is unidirectional, we find for the drag current,

$$
I_{\text {drag }} \sim \frac{\Gamma_{L_{1} 0} \Gamma_{R_{1} 0}\left(\Gamma_{L_{2} 0} \partial \Gamma_{R_{2} 0}-\Gamma_{R_{2} 0} \partial \Gamma_{L_{2} 0}\right)}{\Gamma_{L_{2} 0}+\Gamma_{R_{2} 0}} F\left(V_{\text {sd }}\right),
$$

where $F\left(V_{\text {sd }}\right)=V_{\text {sd }}^{2}, \log V_{\text {sd }}$ for cotunneling-only and cotunneling-assisted drag, respectively. The factor in parentheses in the numerator gives the conditions for drag. Notably, the drag is zero if the lead couplings to the drag QD are constant or differ by a multiplicative factor. Furthermore, the direction of the drag current is determined by two factors concerning the lead couplings to the drag QD: (i) their asymmetry, and (ii) their derivatives.

Drag in graphene-based CQDs.-We now proceed to study the drag effect in an idealized version of the graphene-based CQD structure illustrated in Fig 1(c). The QDs are assumed to be connected to bulk graphene leads with linear DOS, $\rho_{\alpha}(\varepsilon)=\left(g_{s} g_{v} / 2 \pi\left(\hbar v_{F}\right)^{2}\right)\left|\varepsilon-E_{\alpha 0}\right|$, which govern the energy dependence of the lead couplings, i.e., $\Gamma_{\alpha}(\varepsilon)=2 \pi \rho_{\alpha}(\varepsilon)\left|t_{\alpha}\right|^{2}$, where $t_{\alpha}$ is constant, and where the positions of the Dirac points, $E_{\alpha 0}=-e V_{\alpha}$, are controlled by local gates [see Fig. 3(a)]. This allows us to tune both the strength of the lead couplings, $\Gamma_{\alpha 0} \propto\left|\mu_{0}-E_{\alpha 0}\right|$, as well as their derivatives, $\partial \Gamma_{\alpha} \gtrless 0$ on the upper or lower Dirac cones. In order to meet the conditions for a nonzero drag current, $E_{L_{2} 0} \neq E_{R_{2} 0}$ like in
Fig. 3(a) is necessary. Asymmetric tunnel couplings alone, $t_{L_{2}} \neq t_{R_{2}} \rightarrow \Gamma_{L_{2}}(\varepsilon) \propto \Gamma_{R_{2}}(\varepsilon)$, is not enough.

In Figs. 2(a) and 2(c) we show the numerically calculated currents through the drive and drag QDs as a function of gate voltages for the situation in Fig. 3(a) and $k_{B} T \ll e V_{\text {sd }}<U_{12}$. The current through the drive QD in Fig. 2(a) is nonzero along the 00,10 and 01,11 degeneracy lines, and the 10,01 degeneracy line where it is dominated by, respectively, sequential tunneling and nonlocal cotunneling. In addition, elastic cotunneling through the drive QD appears as a background in the Coulomb-blockaded regions.

The induced drag current is shown in Fig. 2(c). A finite drag current is observed along the 10,01 degeneracy line where the nonlocal cotunneling channel is open. With the bias applied symmetrically to the drive dot, this is the case for $e\left|V_{2}-V_{1}\right|=\left|\Delta_{10,01}\right|<e V_{\mathrm{sd}} / 2$. Away from the triple points, $\left|\Delta_{10 / 01,00 / 11}\right| \gg e V_{\text {sd }}$, the drag is driven by nonlocal cotunneling only. In the vicinity of the upper (lower) triple point, $\left|\Delta_{01,11}\right| \lesssim e V_{\text {sd }}\left(\left|\Delta_{10,00}\right| \lesssim e V_{\text {sd }}\right)$, the bias on the drive QD opens the $01 \leftrightarrow 11 \quad(10 \leftrightarrow 00)$ transition via sequential tunneling, and the drag changes to cotunneling-assisted drag. This results in an enhanced drag current compared to the cotunneling-only drag.

Figures 2(b) and 2(d) show the bias dependence of the drive and drag currents at the gate voltages marked by dots in Figs. 2(a) and 2(c). In the linear low-bias regime, $e V_{\text {sd }}<k_{B} T, I_{\text {drive }} \propto V_{\text {sd }}$ and $I_{\text {drag }} \propto V_{\text {sd }}^{2}$ for $\left|\Delta_{10,01}\right|<k_{B} T$ (red, yellow, and green dots). The drag current is
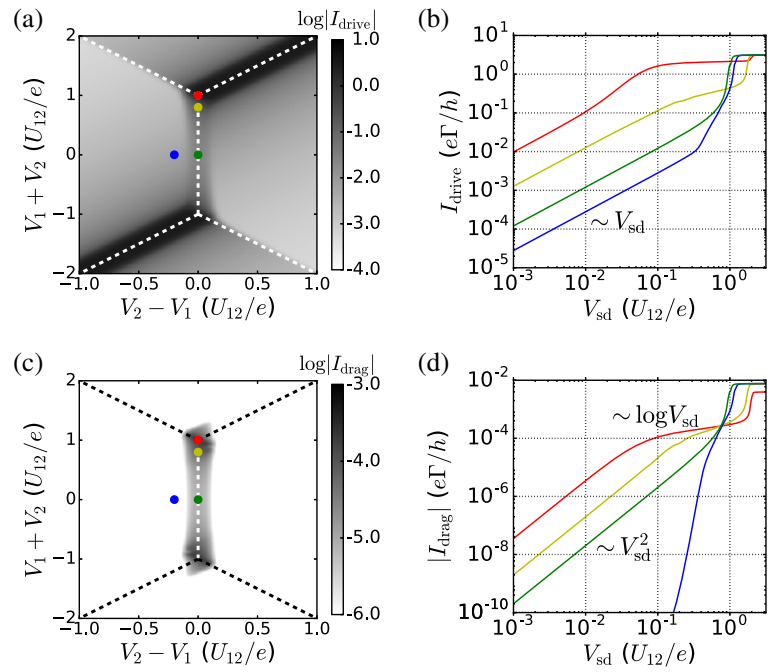

FIG. 2. Drive (top) and drag current (bottom) for the graphenebased CQD in Fig. 1(c), with the voltage configuration in Fig. 3(a). (a),(c) Current vs common gate and gate detuning with a bias voltage $e V_{\mathrm{sd}}=0.2$ applied to the drive QD. (b),(d) Bias dependence of the drive and drag currents at the gate voltages $\left(V_{2}-V_{1}, V_{1}+V_{2}\right)$ marked by dots in the left plots [red: $(0.0,1.0)$, yellow: $(0.0,0.8)$, green: $(0.0,0.0)$, blue: $(-0.2,0.0)]$. Parameters (in units of $U_{12}$ ): $U_{12}=1, \Gamma_{L_{1} 0 / R_{1} 0}=\Gamma_{L_{2} 0 / R_{2} 0}=0.01 \equiv \Gamma$, $\partial \Gamma_{L_{2}}=-\partial \Gamma_{R_{2}}, t_{L_{2}}=t_{R_{2}}, k_{B} T=0.01$. 
(a)

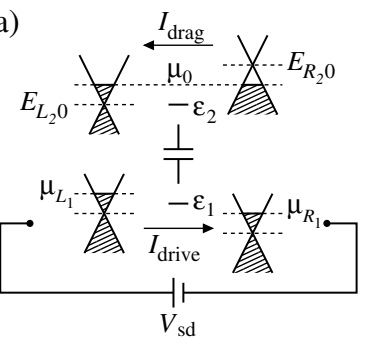

FIG. 3. (a) Energy level diagram of the graphene-based CQD in Fig. 1(c). The QD levels, $\varepsilon_{i}=-e V_{i}$, and the positions of the Dirac points in the leads, $E_{\alpha 0}=-e V_{\alpha}$, are controlled by local gates. (b) Drag current as a function of gate voltage on the leads of the drag system (see Dirac cone insets) at the upper triple point in the stability diagram. Parameters (in units of $U_{12}$ ): $U_{12}=1, \Gamma_{L_{1} 0 / R_{1} 0}=0.01 \equiv \Gamma, \Gamma_{L_{2} 0 / R_{2} 0} \propto$ $\left|\mu_{0}-E_{L_{2} 0 / R_{2} 0}\right|, \partial \Gamma_{L_{2} / R_{2}}=\operatorname{sgn}\left(\mu_{0}-E_{L_{2} 0 / R_{2} 0}\right), t_{L_{2}}=t_{R_{2}}, e V_{\mathrm{sd}}=$ $0.1, k_{B} T=0.01$.

linear in $V_{\text {sd }}$ only at bias voltages $e V_{\text {sd }} \ll k_{B} T$ (not shown). For $\left|\Delta_{10,01}\right|>k_{B} T$ (blue dot), nonlocal cotunneling is exponentially suppressed, $\Gamma_{10,01} \sim e^{-\Delta_{10,01} / k_{B} T}$, resulting in a vanishing drag current. The drive current, however, remains finite due to elastic cotunneling. In the nonlinear regime, $e V_{\text {sd }}>k_{B} T, I_{\text {drag }} \sim V_{\text {sd }}^{2}$ up to $e V_{\text {sd }} \sim$ $\max \left(2\left|\Delta_{10,01}\right|,\left|\Delta_{10 / 01,11}\right|\right)$ where it experiences a crossover to a $I_{\text {drag }} \sim \log V_{\text {sd }}$ dependence in agreement with Eq. (10). At even higher bias, $e V_{\text {sd }} \gtrsim U_{12}$, the conventional drag mechanism [25], which is driven by sequential tunneling, takes over (see also below).

From Eq. (10) it is clear that the direction of the drag current depends, in a nontrivial way, on the lead couplings in the drag system. This is demonstrated in Fig. 3, which shows the drag current at the upper triple point as a function of the positions of the Dirac points in the drag leads. At the diagonal we have $\Gamma_{L_{2}}(\varepsilon)=\Gamma_{R_{2}}(\varepsilon)$, and hence the drag vanishes. Off the diagonal, $\Gamma_{L_{2} 0} \neq \Gamma_{R_{2} 0}$ and $\partial \Gamma_{L_{2}}=\partial \Gamma_{R_{2}}$, the factor $\Gamma_{L_{2} 0}-\Gamma_{R_{2} 0}$ governs the sign of the drag current. Upon crossing the Dirac point in one of the leads, the drag changes sign due to an inversion in the sign of the corresponding DOS derivative. Remarkably, the drag becomes independent on $\left|\mu_{0}-E_{L_{2} 0 / R_{2} 0}\right|$ in this case. This follows from the fact that for symmetric tunnel couplings $\partial \Gamma_{L_{2}}=-\partial \Gamma_{R_{2}}$, which leads to a cancellation of the $\Gamma_{L_{2} 0}+\Gamma_{R_{2} 0}$ factors in Eq. (10). For asymmetric tunnel couplings this is not the case. The unconventional sign of the mesoscopic drag, which we have verified also holds for the conventional drag mechanism [25], is in stark contrast to that of the drag in coupled graphene layers [19].

In the bias spectroscopy of the CQDs shown in Fig. 4, distinct fingerprints of nonlocal cotunneling and the drag mechanism can be observed inside the so-called Coulombblockade diamonds, where the sequential tunneling drive and drag currents are suppressed. It shows the drive (top) and drag (bottom) currents at the center of the stability diagram (green dot in Fig. 2) as a function of gate

(a)

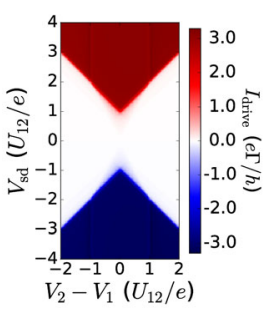

(c)

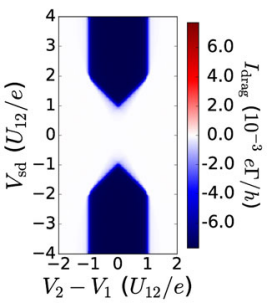

(b)

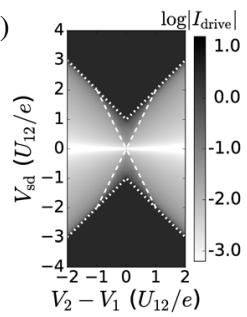

(d)

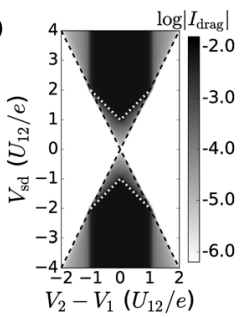

FIG. 4. Bias spectroscopy. The plots show the current through drive (top) and drag (bottom) QDs at the center of the 10,01 degeneracy line with the bias applied to the drive QD. (a), (c) Linear scale. (b),(d) Log scale. The dashed (dotted) lines mark the boundaries to the regions where the currents are dominated by nonlocal cotunneling (sequential tunneling). See Fig. 2 for parameters.

detuning and drive bias. In the low-bias Coulombblockaded regime, $e\left|V_{\mathrm{sd}}\right|<\min \left(U_{12}+e\left|V_{2}-V_{1}\right|, 2 U_{12}\right)$, nonlocal cotunneling manifests itself in nonzero drive and drag currents in the region $\left|V_{\text {sd }}\right| / 2>\left|V_{2}-V_{1}\right|$, which at $\Delta_{10,01}=0$ extends down to zero bias.

At high bias, $e\left|V_{\text {sd }}\right|>U_{12}+e\left|V_{2}-V_{1}\right|$, sequential tunneling dominates both the drive and drag currents. However, for $e\left|V_{2}-V_{1}\right|>U_{12}$, where the conventional drag mechanism [25] is suppressed, cotunneling-assisted drag extends the region with nonzero drag to $e\left|V_{2}-V_{1}\right|<e\left|V_{\mathrm{sd}}\right| / 2$. The different slopes $s$ of the boundaries to the regions where, respectively, sequential tunneling (dotted, $|s|=1$ ) and nonlocal cotunneling (dashed, $|s|=2$ ) dominate the drive and drag currents (see log plots in Fig. 4), is a direct fingerprint of nonlocal cotunneling and its associated drag mechanism [54].

Finally, we estimate the magnitude of the drag current and comment on its experimental verification. Taking $\Gamma_{\alpha}, k_{B} T \sim 0.1 U_{12}$, a drag current of the order of $I_{\mathrm{drag}} \gtrsim$ $\left(U_{12} / \mathrm{meV}\right)^{2} \mathrm{pA}$ is predicted for the cotunneling-assisted drag at $e V_{\text {sd }} \gtrsim k_{B} T, \max \left(2\left|\Delta_{10,01}\right|,\left|\Delta_{10 / 01,00 / 11}\right|\right)$. This is well within experimentally detectable currents and allows for a unique identification of the nonlocal cotunnelingdriven drag via its distinct identifiers-i.e., the bias dependence in Eq. (10) and its fingerprints in bias spectroscopy [Fig. 4(d)]. While the high-bias cotunneling broadening of the drag region in Fig. 4(d) was recently observed in Ref. [33], the drag at low bias remains unexplored.

Conclusions. - In summary, we have identified a ratchetlike drag mechanism in CQDs, driven by nonlocal cotunneling processes. The key ingredient for the drag 
mechanism is that the coupling to the leads be energy dependent. This can be achieved via, e.g., gate-dependent tunnel barriers [37,55], or be an intrinsic property like in graphene-based QD structures with built-in graphene leads [33]. Studying the Coulomb drag in an idealized version of such a QD structure, we demonstrated its nontrivial dependence on the lead couplings and identified its fingerprints in bias spectroscopy. Possible routes for future explorations of drag in CQDs include shot noise and cross correlations characteristics [25,56,57], the effect of level broadening [58,59] and Kondo physics [60,61], which become important at $\Gamma_{\alpha}>k_{B} T$, as well as drag due to other coupling mechanisms between the QDs [62].

We would like to thank J. Santos and N. A. Mortensen for fruitful discussions, and K. Ensslin and D. Bischoff for clarifications on the experimental details in Ref. [33] and comments on the manuscript. The Center for Nanostructured Graphene (CNG) is sponsored by the Danish Research Foundation, Project DNRF103.

Note added.-While this work was under review, we became aware of a related experimental work in which evidence of the nonlocal cotunneling drag mechanism was observed at low bias in bias spectroscopy [63].

*cosby@fys.ku.dk

[1] A. G. Rojo, Electron-drag effects in coupled electron systems, J. Phys. Condens. Matter 11, R31 (1999).

[2] B. N. Narozhny and A. Levchenko, Coulomb drag, arXiv: 1505.07468 .

[3] P. M. Solomon, P. J. Price, D. J. Frank, and D. C. La Tulipe, New Phenomena in Coupled Transport between 2D and 3D Electron-Gas Layers, Phys. Rev. Lett. 63, 2508 (1989).

[4] T. J. Gramila, J.P. Eisenstein, A. H. MacDonald, L. N. Pfeiffer, and K. W. West, Mutual Friction between Parallel Two-Dimensional Electron Systems, Phys. Rev. Lett. 66, 1216 (1991).

[5] U. Sivan, P. M. Solomon, and H. Shtrikman, Coupled Electron-Hole Transport, Phys. Rev. Lett. 68, 1196 (1992).

[6] A.-P. Jauho and H. Smith, Coulomb drag between parallel two-dimensional electron systems, Phys. Rev. B 47, 4420 (1993).

[7] K. Flensberg and B. Yu-Kuang Hu, Coulomb Drag as a Probe of Coupled Plasmon Modes in Parallel Quantum Wells, Phys. Rev. Lett. 73, 3572 (1994).

[8] K. Flensberg, B. Yu-Kuang Hu, A.-P. Jauho, and J. M. Kinaret, Linear-response theory of Coulomb drag in coupled electron systems, Phys. Rev. B 52, 14761 (1995).

[9] A. Kamenev and Y. Oreg, Coulomb drag in normal metals and superconductors: Diagrammatic approach, Phys. Rev. B 52, 7516 (1995).

[10] N. A. Mortensen, K. Flensberg, and A.-P. Jauho, Mesoscopic fluctuations of Coulomb drag between quasiballistic one-dimensional wires, Phys. Rev. B 65, 085317 (2002).
[11] M. Yamamoto, M. Stopa, Y. Tokura, Y. Hirayama, and S. Tarucha, Negative Coulomb drag in a one-dimensional wire, Science 313, 204 (2006).

[12] D. Laroche, G. Gervais, M. P. Lilly, and J. L. Reno, Positive and negative Coulomb drag in vertically integrated onedimensional quantum wires, Nat. Nanotechnol. 6, 793 (2011).

[13] D. Laroche, G. Gervais, M. P. Lilly, and J. L. Reno, 1D-1D Coulomb drag signature of a Luttinger liquid, Science $\mathbf{3 4 3}$, 631 (2014).

[14] A. A. Shylau and A.-P. Jauho, Plasmon-mediated Coulomb drag between graphene waveguides, Phys. Rev. B 89, 165421 (2014).

[15] W.-K. Tse, B. Yu-Kuang Hu, and S. Das Sarma, Theory of Coulomb drag in graphene, Phys. Rev. B 76, 081401(R) (2007).

[16] E. H. Hwang, R. Sensarma, and S. Das Sarma, Coulomb drag in monolayer and bilayer graphene, Phys. Rev. B 84, 245441 (2011).

[17] S. Kim, I. Jo, J. Nah, Z. Yao, S. K. Banerjee, and E. Tutuc, Coulomb drag of massless fermions in graphene, Phys. Rev. B 83, 161401(R) (2011).

[18] M. I. Katsnelson, Coulomb drag in graphene single layers separated by a thin spacer, Phys. Rev. B 84, 041407(R) (2011).

[19] R. V. Gorbachev, A. K. Geim, M. I. Katsnelson, K. S. Novoselov, T. Tudorovskiy, I. V. Grigorieva, A. H. MacDonald, S. V. Morozov, K. Watanabe, T. Taniguchi, and L. A. Ponomarenko, Strong Coulomb Drag and Broken Symmetry in Double-Layer Graphene, Nat. Phys. 8, 896 (2012).

[20] J. C. W. Song, D. A. Abanin, and L. S. Levitov, Coulomb drag mechanisms in graphene, Nano Lett. 13, 3631 (2013).

[21] N. A. Mortensen, K. Flensberg, and A.-P. Jauho, Coulomb Drag in Coherent Mesoscopic Systems, Phys. Rev. Lett. 86, 1841 (2001).

[22] M. E. Raikh and F. von Oppen, Coulomb Drag for Strongly Localized Electrons: A Pumping Mechanism, Phys. Rev. Lett. 89, 106601 (2002).

[23] Q.-f. Sun, H. Guo, and J. Wang, Correlated two-electron transport: A principle for a charge pump, Phys. Rev. B 68, 035318 (2003).

[24] A. Levchenko and A. Kamenev, Coulomb Drag in Quantum Circuits, Phys. Rev. Lett. 101, 216806 (2008).

[25] R. Sánchez, R. López, D. Sánchez, and M. Büttiker, Mesoscopic Coulomb Drag, Broken Detailed Balance, and Fluctuation Relations, Phys. Rev. Lett. 104, 076801 (2010)

[26] A. A. Clerk, M. H. Devoret, S. M. Girvin, F. Marquardt, and R. J. Schoelkopf, Introduction to quantum noise, measurement, and amplification, Rev. Mod. Phys. 82, 1155 (2010).

[27] R. Aguado and L. P. Kouwenhoven, Double Quantum Dots as Detectors of High-Frequency Quantum Noise in Mesoscopic Conductors, Phys. Rev. Lett. 84, 1986 (2000).

[28] E. Onac, F. Balestro, L. H. Willems van Beveren, U. Hartmann, Y. V. Nazarov, and L. P. Kouwenhoven, Using a Quantum Dot as a High-Frequency Shot Noise Detector, Phys. Rev. Lett. 96, 176601 (2006).

[29] S. Gustavsson, M. Studer, R. Leturcq, T. Ihn, K. Ensslin, D. C. Driscoll, and A.C. Gossard, Frequency-Selective Single-Photon Detection Using a Double Quantum Dot, Phys. Rev. Lett. 99, 206804 (2007). 
[30] V. S. Khrapai, S. Ludwig, J. P. Kotthaus, H. P. Tranitz, and W. Wegscheider, Double-Dot Quantum Ratchet Driven by an Independently Biased Quantum Point Contact, Phys. Rev. Lett. 97, 176803 (2006).

[31] D. Harbusch, D. Taubert, H. P. Tranitz, W. Wegscheider, and S. Ludwig, Phonon-Mediated versus Coulombic Backaction in Quantum Dot Circuits, Phys. Rev. Lett. 104, 196801 (2010).

[32] G. Shinkai, T. Hayashi, T. Ota, K. Muraki, and T. Fujisawa, Bidirectional Current drag induced by two-electron cotunneling in coupled double quantum dots, Appl. Phys. Express 2, 081101 (2009).

[33] D. Bischoff, M. Eich, O. Zilberberg, C. Rössler, T. Ihn, and K. Ensslin, Measurement back-action in stacked graphene quantum dots, Nano Lett. 15, 6003 (2015).

[34] C. Volk, S. Engels, C. Neumann, and C. Stampfer, Back action of graphene charge detectors on graphene and carbon nanotube quantum dots, Phys. Status Solidi B 252, 2461 (2015).

[35] R. Sánchez and M. Büttiker, Optimal energy quanta to current conversion, Phys. Rev. B 83, 085428 (2011).

[36] B. Sothmann, R. Sánchez, and A. N. Jordan, Thermoelectric energy harvesting with quantum dots, Nanotechnology 26, 032001 (2015).

[37] H. Thierschmann, R. Sánchez, B. Sothmann, F. Arnold, C. Heyn, W. Hansen, H. Buhmann, and L. W. Molenkamp, Three-terminal energy harvester with coupled quantum dots, Nat. Nanotechnol. 10, 854 (2015).

[38] J. V. Koski, A. Kutvonen, I. M. Khaymovich, T. Ala-Nissila, and J. P. Pekola, On-Chip Maxwell's Demon as an Information-Powered Refrigerator, Phys. Rev. Lett. 115, 260602 (2015).

[39] W. G. van der Wiel, S. De Franceschi, J. M. Elzerman, T. Fujisawa, S. Tarucha, and L. P. Kouwenhoven, Electron transport through double quantum dots, Rev. Mod. Phys. 75, 1 (2002).

[40] M. R. Connolly, K. L. Chiu, S. P. Giblin, M. Kataoka, J. D. Fletcher, C. Chua, J. P. Griffiths, G. A. C. Jones, V. I. Fal'ko, C. G. Smith, and T. J. B. M. Janssen, Gigahertz quantized charge pumping in graphene quantum dots, Nat. Nanotechnol. 8, 417 (2013).

[41] L. A. Ponomarenko, F. Schedin, M. I. Katsnelson, R. Yang, E. W. Hill, K. S. Novoselov, and A. K. Geim, Chaotic Dirac billiard in graphene quantum dots, Science 320, 356 (2008).

[42] C. Stampfer, J. Güttinger, F. Molitor, D. Graf, T. Ihn, and K. Ensslin, Tunable Coulomb blockade in nanostructured graphene, Appl. Phys. Lett. 92, 012102 (2008).

[43] L.-J. Wang, G. Cao, T. Tu, H.-O. Li, C. Zhou, X.-J. Hao, Z. Su, G.-C. Guo, G.-P. Guo, and H.-W. Jiang, A graphene quantum dot with a single electron transistor as integrated charge sensor, Appl. Phys. Lett. 97, 262113 (2010).

[44] J. Güttinger, F. Molitor, C. Stampfer, S. Schnez, A. Jacobsen, S. Dröscher, T. Ihn, and K. Ensslin, Transport through graphene quantum dots, Rep. Prog. Phys. 75, 126502 (2012).

[45] D. Bischoff, A. Varlet, P. Simonet, M. Eich, H. C. Overweg, T. Ihn, and K. Ensslin, Localized charge carriers in graphene nanodevices, Appl. Phys. Rev. 2, 031301 (2015).

[46] B. Huard, J. A. Sulpizio, N. Stander, K. Todd, B. Yang, and D. Goldhaber-Gordon, Transport Measurements Across a Tunable Potential Barrier in Graphene, Phys. Rev. Lett. 98, 236803 (2007).

[47] B. Özyilmaz, P. Jarillo-Herrero, D. Efetov, D. A. Abanin, L. S. Levitov, and P. Kim, Electronic Transport and Quantum Hall Effect in Bipolar Graphene $p-n-p$ junctions, Phys. Rev. Lett. 99, 166804 (2007).

[48] H. Bruus and K. Flensberg, Many-body Quantum Theory in Condensed Matter Physics (Oxford University Press, New York, 2004).

[49] D. V. Averin and Yu. V. Nazarov, Virtual Electron Diffusion during Quantum Tunneling of the Electric Charge, Phys. Rev. Lett. 65, 2446 (1990).

[50] A. Furusaki and K. A. Matveev, Theory of strong inelastic cotunneling, Phys. Rev. B 52, 16676 (1995).

[51] S. De Franceschi, S. Sasaki, J. M. Elzerman, W. G. van der Wiel, S. Tarucha, and L. P. Kouwenhoven, Electron Cotunneling in a Semiconductor Quantum Dot, Phys. Rev. Lett. 86, 878 (2001).

[52] M. Turek and K. A. Matveev, Cotunneling thermopower of single electron transistors, Phys. Rev. B 65, 115332 (2002).

[53] J. Koch, F. von Oppen, Y. Oreg, and E. Sela, Thermopower of single-molecule devices, Phys. Rev. B 70, 195107 (2004).

[54] See Supplemental Material at http://link.aps.org/ supplemental/10.1103/PhysRevLett.116.196801, which includes (i) a detailed account of the cotunneling regularization scheme generalized to situations with energy-dependent lead couplings, and (ii) results for the bias spectroscopy with an asymmetrically biased drive dot.

[55] J. Waissman, M. Honig, S. Pecker, A. Benyamini, A. Hamo, and S. Ilani, Realization of pristine and locally tunable one-dimensional electron systems in carbon nanotubes, Nat. Nanotechnol. 8, 569 (2013).

[56] D. T. McClure, L. DiCarlo, Y. Zhang, H.-A. Engel, C. M. Marcus, M. P. Hanson, and A. C. Gossard, Tunable Noise Cross Correlations in a Double Quantum Dot, Phys. Rev. Lett. 98, 056801 (2007).

[57] K. Kaasbjerg and W. Belzig, Full counting statistics and shot noise of cotunneling in quantum dots and singlemolecule transistors, Phys. Rev. B 91, 235413 (2015).

[58] A. Thielmann, M. H. Hettler, J. König, and G. Schön, Cotunneling Current and Shot Noise in Quantum Dots, Phys. Rev. Lett. 95, 146806 (2005).

[59] O. Zilberberg, A. Carmi, and A. Romito, Measuring cotunneling in its wake, Phys. Rev. B 90, 205413 (2014).

[60] S. Amasha, A. J. Keller, I. G. Rau, A. Carmi, J. A. Katine, H. Shtrikman, Y. Oreg, and D. Goldhaber-Gordon, Pseudospin-Resolved Transport Spectroscopy of the Kondo Effect in a Double Quantum Dot, Phys. Rev. Lett. 110, 046604 (2013).

[61] Z.-q. Bao, A.-M. Guo, and Q.-f. Sun, Orbital Kondo effect in a parallel double quantum dot, J. Phys. Condens. Matter 26, 435301 (2014).

[62] G.-W. Deng, D. Wei, S.-X. Li, J. R. Johansson, W.-C. Kong, H.-O. Li, G. Cao, M. Xiao, G.-C. Guo, F. Nori, H.-W. Jiang, and G.-P. Guo, Coupling two distant double quantum dots with a microwave resonator, Nano Lett. 15, 6620 (2015).

[63] A. J. Keller, J. S Lim, D. Sánchez, R. López, S. Amasha, J. A. Katine, H. Shtrikman, and D. Goldhaber-Gordon, Cotunneling drag effect in Coulomb-coupled quantum dots, arXiv:1603.00799. 